\title{
Supporting information: Asymmetric GaN/ZnO engineered resistive memory device for electronic synapses
}

Muhammad Umair Khan ${ }^{\dagger a, b}$, Chaudhry Muhammad Furqan ${ }^{\dagger b, c}$, Jungmin Kim ${ }^{\dagger a}$, Sobia Ali Khan ${ }^{\mathrm{d}}$, Qazi Muhammad Saqib ${ }^{\mathrm{a}}$, Mahesh Y. Chougale ${ }^{\mathrm{a}}$, Rayyan Ali Shaukat ${ }^{\mathrm{a}}$, Moon Hee Kang ${ }^{\mathrm{d}}$, Nobuhiko P. Kobayashie, Jinho Bae ${ }^{\mathrm{a} *}$, and Hoi-Sing Kwok ${ }^{\mathrm{b}, \mathrm{c} *}$

$\uparrow$ All authors are considered as the first contributing authors of this manuscript

${ }^{a}$ Department of Ocean System Engineering, Jeju National University, 102 Jejudaehakro, Jeju 63243, Republic of Korea.

bDepartment of Electronic and Computer Engineering, The Hong Kong University of Science and Technology, Clear Water Bay, Kowloon, Hong Kong

'State Key Laboratory on Advanced Displays and Optoelectronics Technologies, The Hong Kong University of Science and Technology, Clear Water Bay, Kowloon, Hong Kong

dSchool of Electronics Engineering, Chungbuk National University, Cheongju 28644, South Korea 'Baskin School of Engineering, University of California Santa Cruz, 1156 High Street, Santa Cruz, CA, 95064, USA

*Email: baejh@jejunu.ac.kr, eekwok@ust.hk 


\section{Table of contents}

\section{Figures}

Figure S1. (a) SEM image, and (b) 3D nano profile of top electrode. The surface morphology of (c) GaN and (d) $\mathrm{ZnO}$. The 3D AFM image to investigate the roughness of (e) $\mathrm{ZnO}$ and (f) $\mathrm{GaN}$.

Figure S2. XPS of ITO/ZnO/GaN/Ag.

Figure S3. (a) I-V curve and (b) Semi log I-V curve of ITO/GaN/Ag. (c) I-V endurance cycles of 100 times and (d) device endurance between HRS and LRS. TSCCLC mechanism showing (e) positive and (f) negative voltage region.

Figure S4. (a) $2 \times 2$ crossbar array. (b) Sneak current parameters on positive and negative read voltage. (c) Symmetric sneak current simulation. (d) Asymmetric sneak current simulation.

Figure S5. LTP and LTP at voltage point $1.5 \mathrm{~V}$ and $-1.5 \mathrm{~V}$, respectively.

Figure 56. (a) $2 \times 2$ crossbar array. (b) Kernel density estimation probability and histogram of asymmetric. (c) Kernel density estimation probability and histogram of symmetric neuromorphic device. (d) Kernel density estimation probability of asymmetric sneak current and symmetric sneak current (where, we can see that the different current states from HRS1 to HRS5.).

\section{Tables}

Table S1. This work is compared with $\mathrm{ZnO}$ based asymmetric resistive memory devices. 


\section{Device surface characterization:}

\section{Figure S1.}
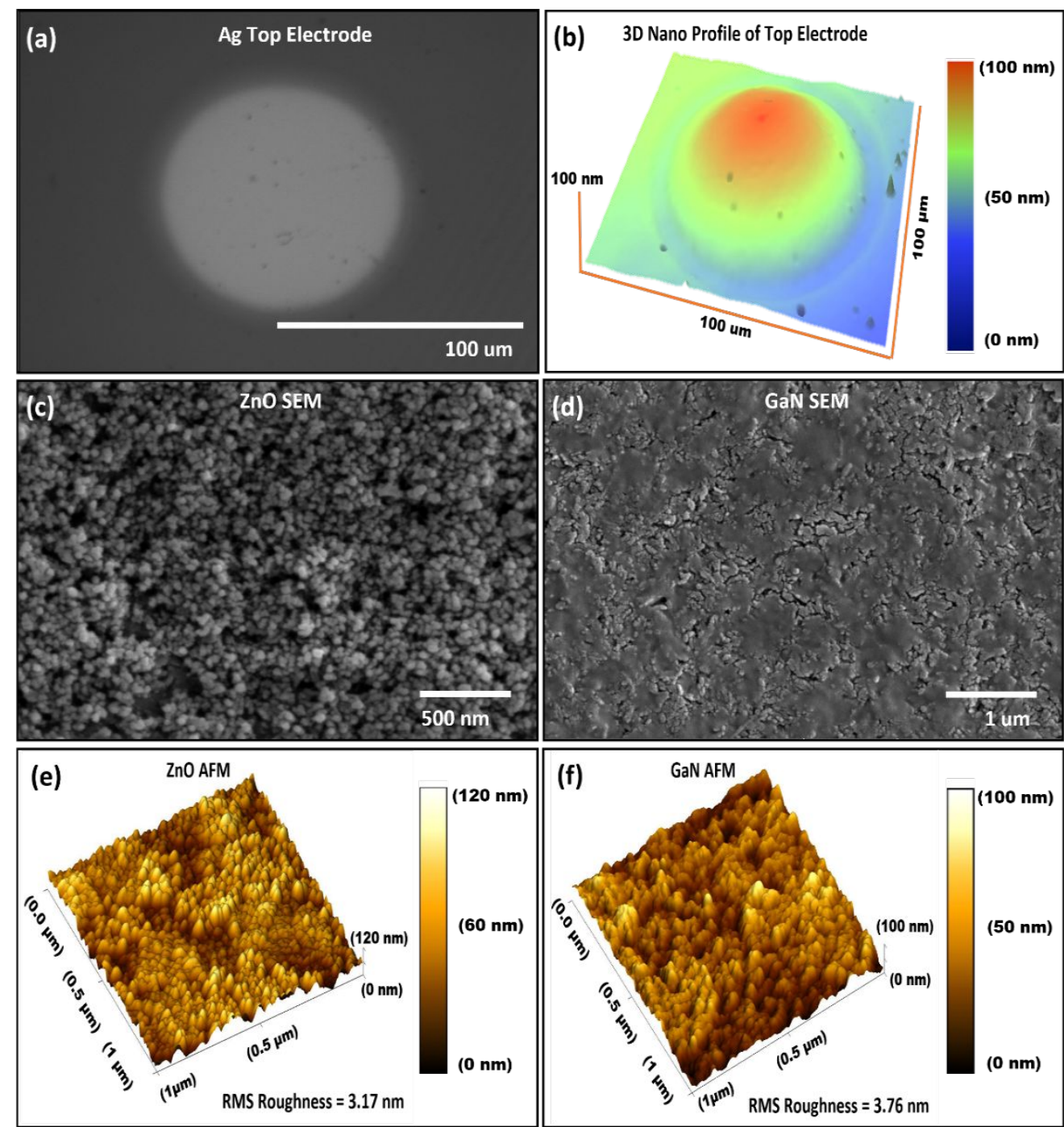

Figure S1. (a) SEM image and (b) 3D nano profile of top electrode. The surface morphology of (c) GaN and (d) $\mathrm{ZnO}$. The 3D AFM image to investigate the roughness of (e) $\mathrm{ZnO}$ and (f) $\mathrm{GaN}$.

The top Ag electrode is patterned using a $100 \mu \mathrm{m}$ shadow mask as shown in Figure S1a and the height profile is measured using a 3D nano profiler, which is approximate $\sim 90 \mathrm{~nm}$ as shown in Figure S1b. The surface morphology of $\mathrm{ZnO}$ is shown in Figure $\mathrm{S} 1 \mathrm{c}$ and $\mathrm{GaN}$ as shown in Figure $\mathrm{S} 1 \mathrm{~d}$ is analyzed at magnification levels of $500 \mathrm{~nm}$ and $1 \mathrm{um}$, respectively. 3D topographical AFM images of $\mathrm{ZnO}$ as shown in Figure $\mathrm{S} 1 \mathrm{e}$ and GaN as shown in Figure S1f, visibility of atomic traces with the estimated Root Mean Square (RMS) roughness of 3.17 $\mathrm{nm}$ and $3.76 \mathrm{~nm}$, respectively were calculated in the scanning area of $1 \mu \mathrm{m} \times 1 \mu \mathrm{m}$. 
XPS:

Figure S2.

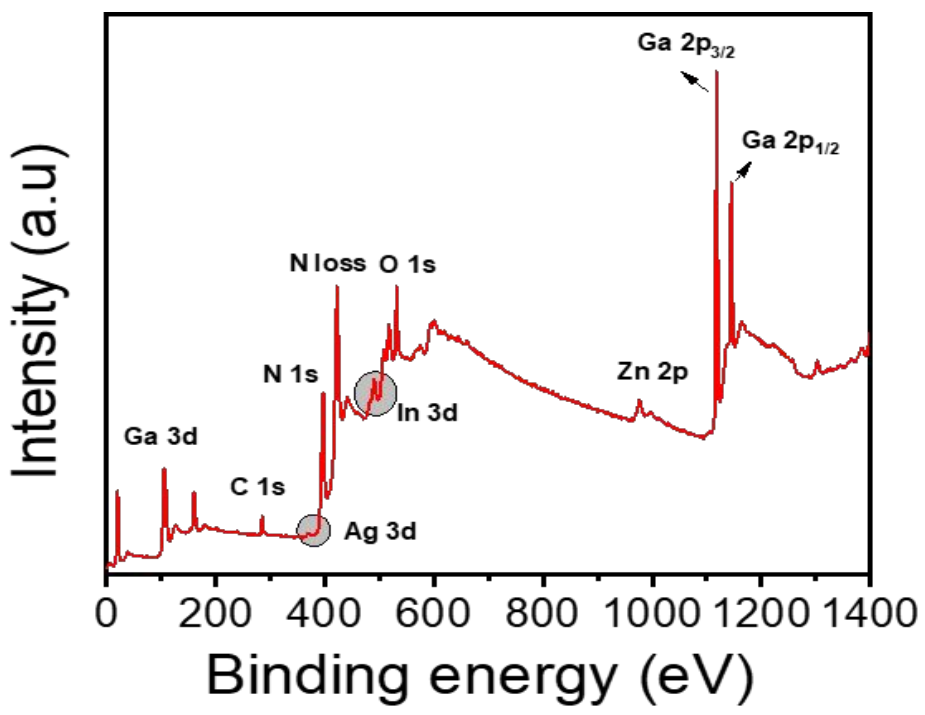

Figure S2. XPS of ITO/ZnO/GaN/Ag. 
Figure S3.
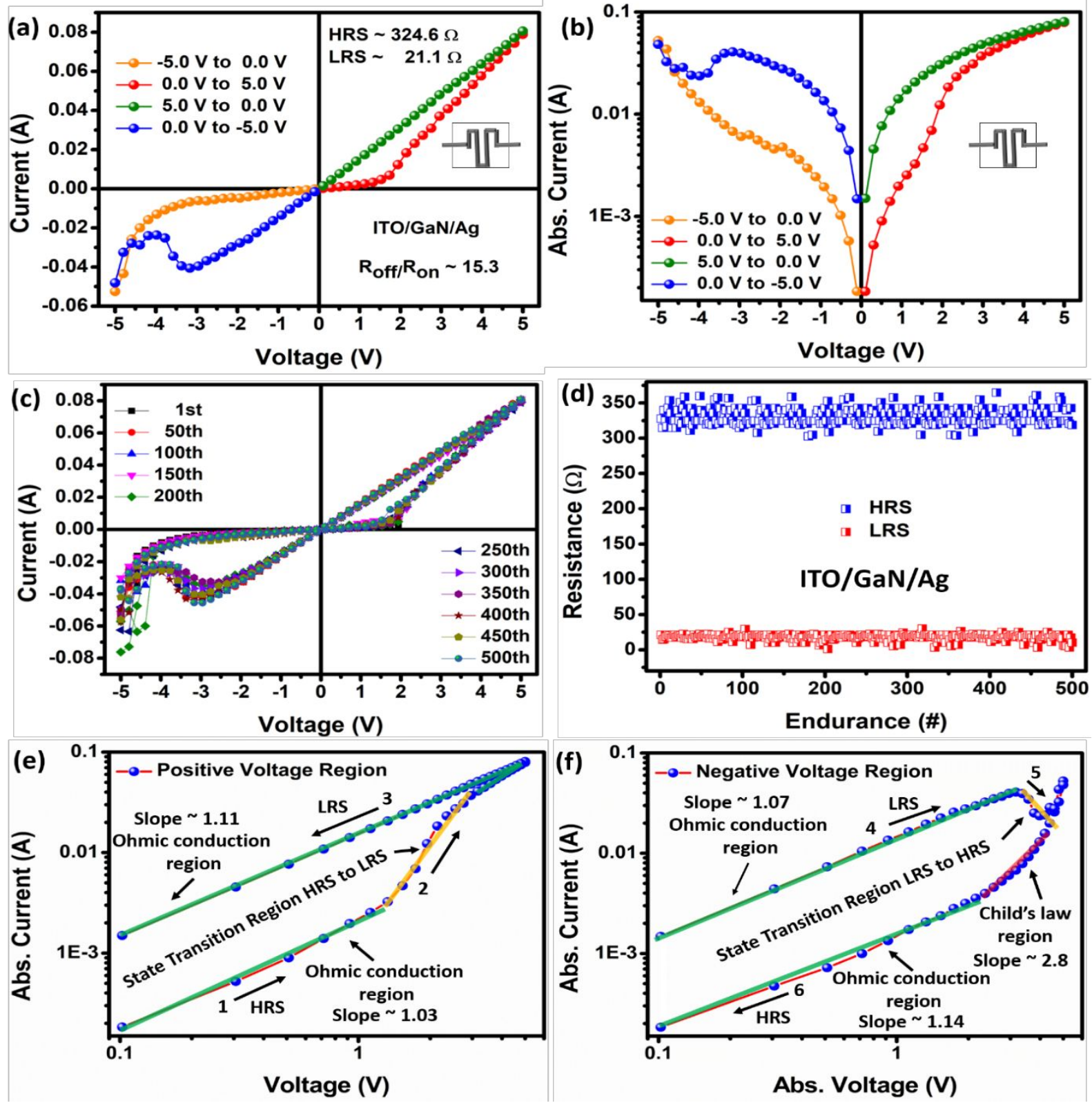

Abs. Voltage (V)

Figure S3. (a) I-V curve and (b) Semi log I-V curve of ITO/GaN/Ag. (c) I-V endurance cycles of 100 times and (d) device endurance between HRS and LRS. TSCCLC mechanism showing (e) positive and (f) negative voltage region.

To characterize ITO/GaN/Ag device, dual sweep voltage of $\pm 5 \mathrm{~V}$ was applied, in which ITO and Ag used as bottom and top electrode and $\mathrm{GaN}$ as an active layer, as result we achieved stable bipolar resistive switching behavior as shown in Figure S3a, and semi-log I-V graph is shown in Figure S3b with HRS $326.6 \Omega$ and LRS 
$21.1 \Omega$, with $\mathrm{R}_{\text {off }} / \mathrm{R}_{\text {on }}$ ratio $\sim 15$. The bipolar switching cycle repeatability for 500 cycles is shown in Figure $3 \mathrm{c}$ and device endurance between HRS and LRS is shown in Figure S3d. The conduction mechanism of the ITO/GaN/Ag device follows a trap-controlled space charge limited current (TCSCLC) model as shown in Figure S3e and $\mathrm{f}$. The HRS governs by ohmic conduction $(\mathrm{I} \propto \mathrm{V})$ green marked region due to thermally generality charge carries with slope $\sim 1$ and child's law which is a red marked region, in which trap charges increase with applied voltage showing slope $\sim 2$. In-state transition region exponential increase $\left(\mathrm{I} \propto \mathrm{V}^{\mathrm{m}}\right)$ in current due to the maximum number of charge carriers, which helps to a shift memory device from HRS to LRS (orange marked region). The LRS dominantly operates in the ohmic conduction region, due to the presence of free charge carriers with slope $\sim 1$ (green marked region). The ITO/GaN/Ag memory device shows very high current conduction, and it should be minimized. 


\section{Figure S4.}
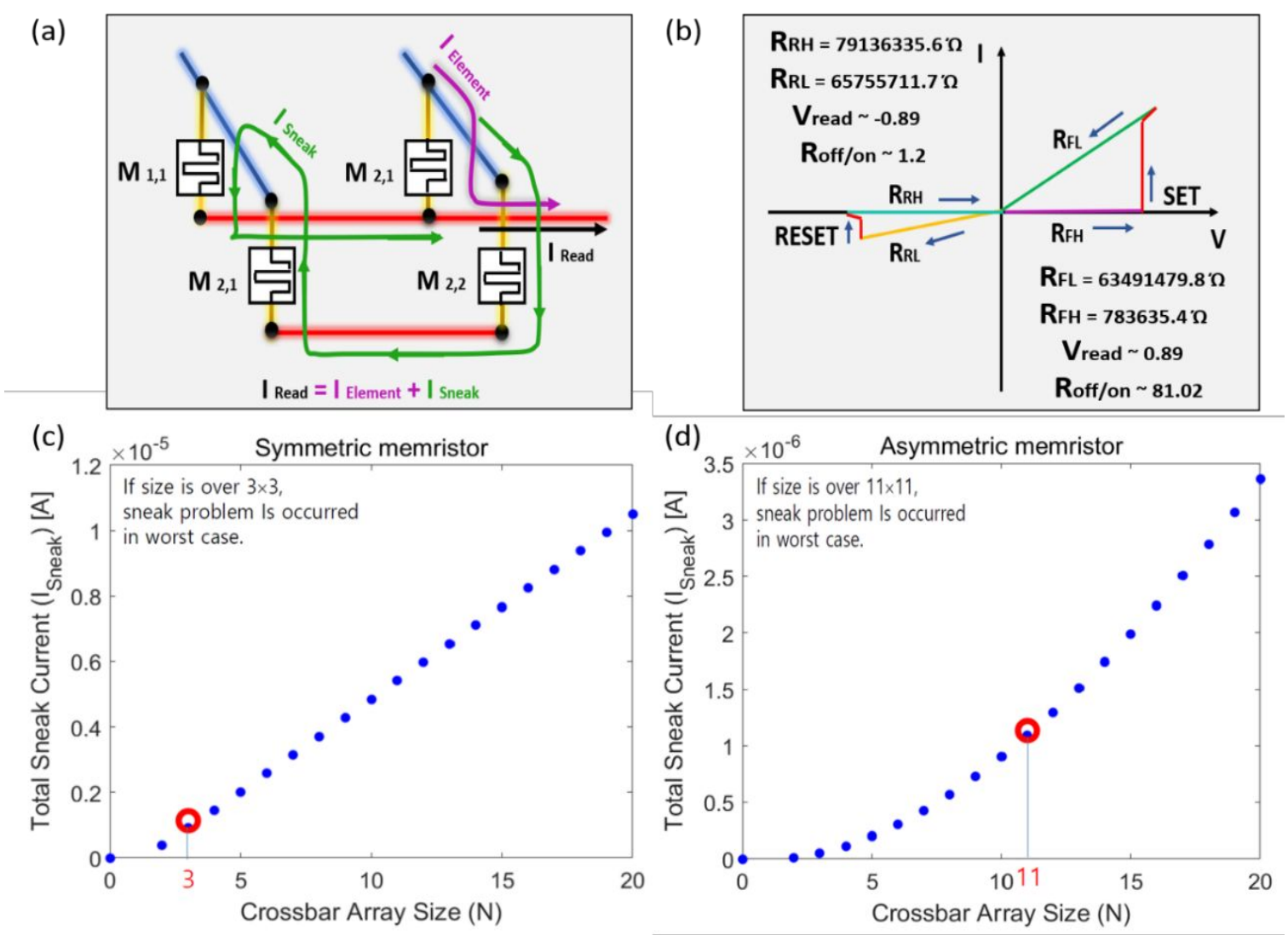

Figure S4. (a) $2 \times 2$ crossbar array. (b) Sneak current parameters on positive and negative read voltage. (c) Symmetric sneak current simulation. (d) Asymmetric sneak current simulation.

Figure S4a shows the sneak current model based on a $2 \times 2$ crossbar array for understanding, in which $\mathrm{e}_{\text {lement }}$ is a read current from memristor, $\mathrm{I}_{\text {sneak }}$ is the current from the sneak path. The $\mathrm{I}_{\text {read }}$ current is the sum of $\mathrm{I}_{\text {Element }}$ and $\mathrm{I}_{\text {sneak. }}$ For the crossbar array $\mathrm{I}_{\text {sneak }}{ }^{1}$. Should be minimized for large-scale integration a shown in Figure S4b. To investigative the sneak current in ITO/ZnO/GaN/Ag device, we have checked the forward current high and low resistance as $\mathrm{R}_{\mathrm{FH}}$ and $\mathrm{R}_{\mathrm{FL}} \sim 63.49 \mathrm{M} \Omega$ and $0.78 \mathrm{M} \Omega$, respectively at a read voltage of $\sim 0.89 \mathrm{~V}$ and reversed current high resistance and low resistance as $\mathrm{R}_{\mathrm{RH}}$ and $\mathrm{R}_{\mathrm{RL}} \sim 79.13 \mathrm{M} \Omega$ and $65.75 \mathrm{M} \Omega$, respectively at a read voltage of $\sim-0.89 \mathrm{~V}$ a shown in Figure 4c. The asymmetric resistive memory device as shown in Figure S4d can be used to block cross talk in an $11 \times 11$ crossbar array. In bipolar case resistive memory device can only be used to block sneak current in $3 \times 3$ crossbar array. 
Comparison of asymmetric resistive memories:

Table S1.

Table S1. This work is compared with $\mathrm{ZnO}$ based asymmetric resistive memory devices.

\begin{tabular}{|c|c|c|c|c|c|}
\hline Number & Device structure & $\begin{array}{c}\text { Endurance } \\
\text { cycles }\end{array}$ & $\begin{array}{c}\text { Retention } \\
\text { time }\end{array}$ & $\begin{array}{c}\mathbf{R}_{\text {off } / \text { on }} \\
\text { Ratio }\end{array}$ & References \\
\hline $\mathbf{1}$ & $\mathrm{FTO} / \mathrm{ZnO} / \mathrm{Al}$ & 10,000 & $1000 \mathrm{sec}$ & 100 & 1 \\
\hline $\mathbf{2}$ & $\mathrm{ITO} / \mathrm{ZnO}: \mathrm{Ga} / \mathrm{ZnO} / \mathrm{ZnO}: \mathrm{Li} / \mathrm{Ag}$ & 1000 & $10^{4} \mathrm{sec}$ & 100 & 2 \\
\hline $\mathbf{3}$ & $\mathrm{Pt} / \mathrm{ZnO} / \mathrm{ZnO} / \mathrm{Ag}$ & 30 & & 40 & 3 \\
\hline $\mathbf{4}$ & $\mathrm{ITO} / \mathrm{ZnO} / \mathrm{GaN} / \mathrm{Ag}$ & 500 & & 81 & This work \\
\hline
\end{tabular}


Simulation parameters of LTP and LTD: To calculate sneak current model in a $2 \times 2$ crossbar array for the asymmetric and symmetric neuromorphic device:

Figure S5.

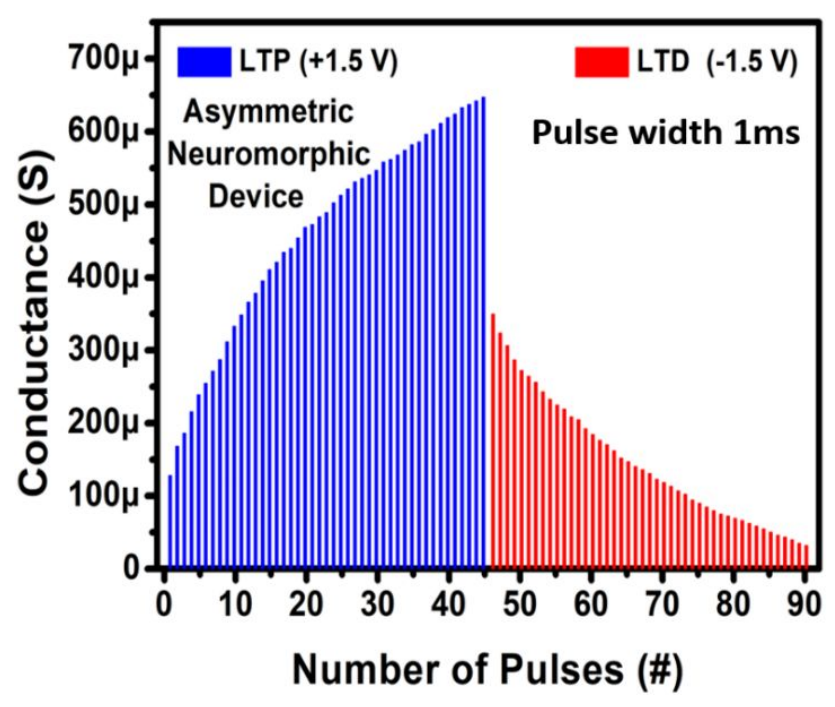

Figure S5. LTP and LTP at voltage point $1.5 \mathrm{~V}$ and $-1.5 \mathrm{~V}$, respectively.

The LTP and LTD at $\pm 1.5 \mathrm{~V}$ as shown in Figure S5 is extracted from device shown in main manuscript Figure 4. For symmetric neuromorphic device LTP data at $+1.5 \mathrm{~V}$ data is shown in Figure S5 is extracted from main manuscript Figure 4, however LTD data at $-1.5 \mathrm{~V}$ is considered as same as LTP for symmetric neuromorphic device. This data is used to perform the simulation of $2 \times 2$ crossbar array to compare the crosstalk effect in one directional (asymmetric) and symmetric neuromorphic device as shown in Figure S6. 
Figure S6.

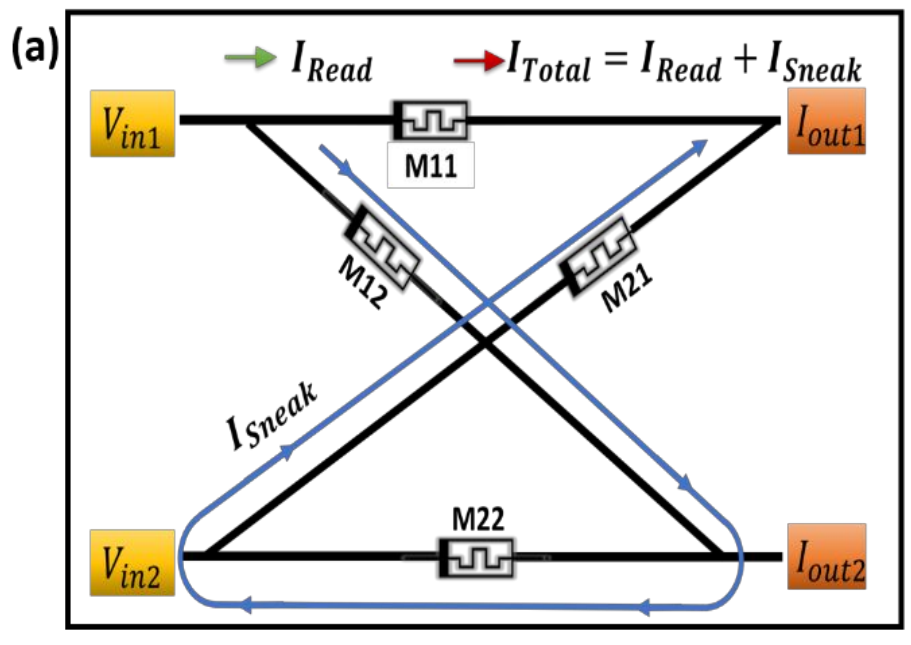

2×2 Crossbar Array

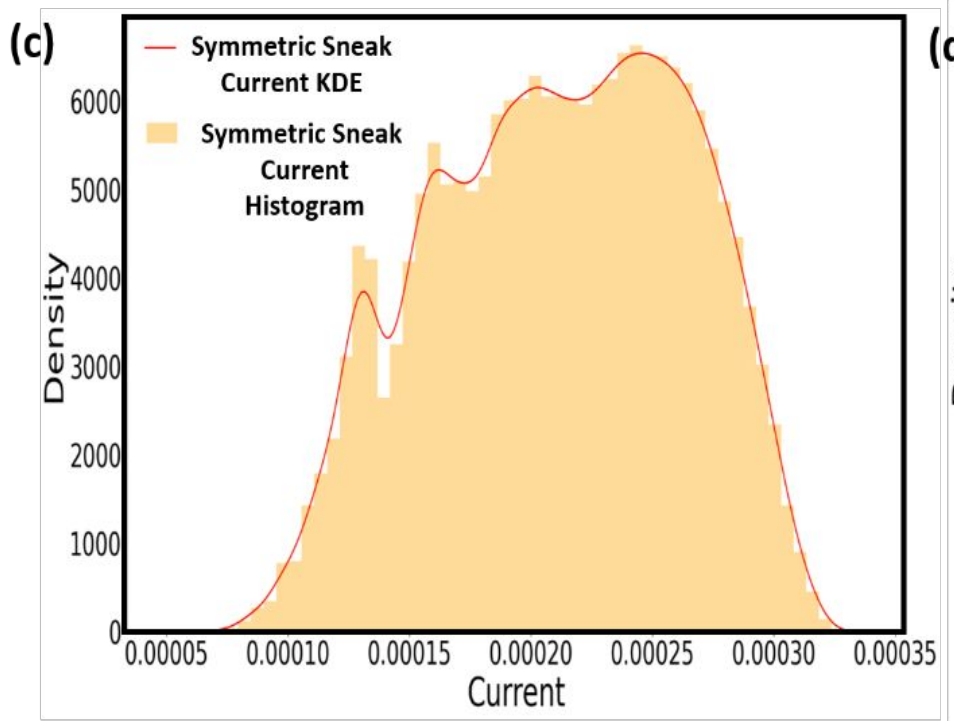

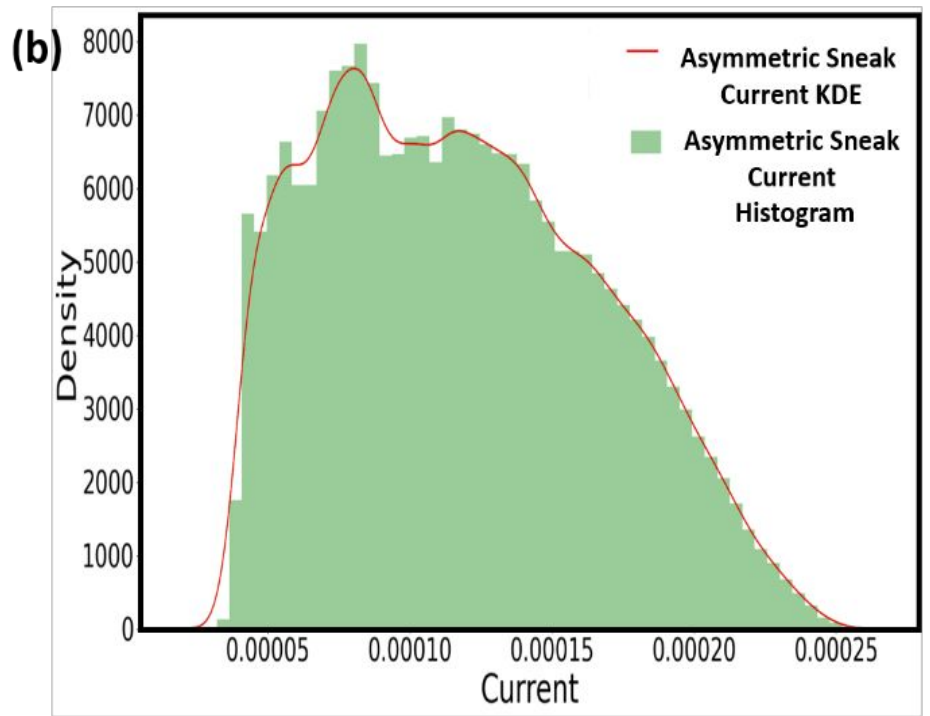

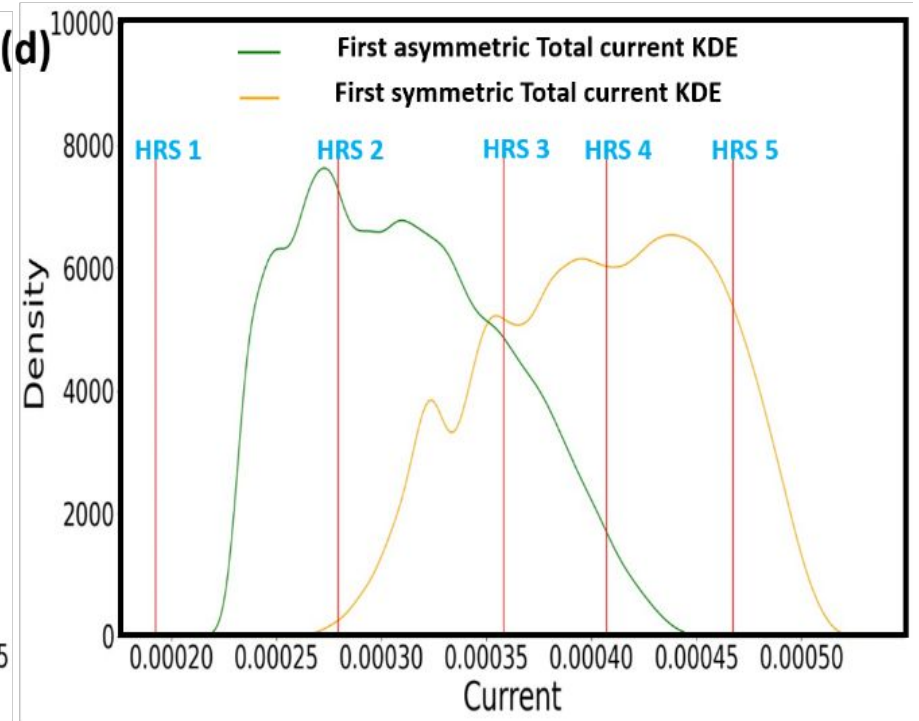

Figure S6. (a) $2 \times 2$ crossbar array. (b) Kernel density estimation probability and histogram of asymmetric. (c) Kernel density estimation probability and histogram of symmetric neuromorphic device. (d) Kernel density estimation probability of asymmetric sneak current and symmetric sneak current (where, we can see that the different current states from HRS1 to HRS5.).

To evaluate the sneak current problem for the proposed neuromorphic device in a crossbar array, it demonstrates a neural network model based on a $2 \times 2$ array as shown in Figure $\mathrm{S} 6 \mathrm{a}$, where $\mathrm{I}_{\text {Read }}$ is a true current for reading memristor and $I_{\text {sneak }}$ is a leakage sneak current if $V_{\text {in1 }} \neq 0$ and $V_{\text {in2 }}=0$ for a clear situation and a simple 
consideration. Here, total current $\left(\mathrm{I}_{\text {Total }}\right)$ is the sum of $\mathrm{I}_{\text {Read }}$ and $\mathrm{I}_{\text {Sneak }}$ at output. In this simple network circuit, the measure total current is measured as bellow Eq. 1.

$$
I_{\text {Total }}=I_{\text {Read }}+I_{\text {Sneak }}=\frac{V_{\text {in } 1}}{M_{11}}+\frac{V_{\text {in } 1}}{M_{12}+M_{21}+M_{22}}
$$

Here, $V_{\text {in } 1}$ is the input voltage and $M_{11}, M_{12}, M_{21}$, and $M_{22}$ are every test memristors value. As a real circuit based on Neuromem devices as shown in Figure S6a, a sneak current path is undesired directional value. In Eq. 1 , the different value of asymmetric and symmetric memristors is $M_{22}$, since it has a reverse directional current path. Hence, reverse high resistance $M_{22}$ of asymmetric resistive switching can help to block an undesired sneak current. Especially, the various resistance states in Figure S6c and d cause to increase the sneak current.

To perform the $2 \times 2$ neuromorphic network, the value of LTP and LTD are obtained to equate sneak current in a crossbar array for the symmetric and asymmetric neuromorphic device as shown in supplementary Figure S5. Here, we set $1.5 \mathrm{~V}$ for $V_{i n 1}$ and $0 \mathrm{~V}$ for $V_{i n 2}$. From Eq. 1, the sneak current $\left(I_{\text {Sneak }}\right)$ profiles can be easily calculated for asymmetric and symmetric memristor device as shown in Figure S7b and c. From these histogram profile probability density functions (pdfs) of asymmetric and symmetric sneak current can estimated as Gaussian kernel density estimation (KDE) as solid lines shown in Figure S6b and c, respectively. Here, the KDE is a nonparametric method of calculating the probability density function (pdf) through Matlab R2019a as

$$
f_{h}(x)=\frac{1}{n} \sum_{i=1}^{n} K_{h}\left(x-x_{i}\right)=\frac{1}{n h} \sum_{i=1}^{n} K_{h}\left(\frac{x-x_{i}}{h}\right)
$$

where, $K$ is a kernel function, $n$ is the number of data, $h$ is a smoothing parameter called the bandwidth and $x$ is given point. In simulation, we use gaussian function for $K, h$ for symmetric and asymmetric neuromorphic device as following 6.03106e-06, 6.60652e-06, respectively. As compared to Figure S6b and c, the bandwidth of the asymmetric pdf is narrower than one of the symmetric pdf, hence asymmetric resistive switching is smaller than the other to detect read current $\left(\mathrm{I}_{\mathrm{Read}}\right)$. In simulation, if we read 1st HRS state, we have to consider a reading error by effecting the sneak current. For example, to calculate misreading error as other HRS states, we consider other 
states from $2^{\text {nd }} H R S(H R S 2)$ state to $5^{\text {th }} H R S(H R S 5)$ state, and their current states are $0.000279381,0.000358304$, 0.000407309, and 0.000467483, respectively. In total current with 1st HRS (HRS1) state, the misreading errors of other states were detected as $100 \%, 77.8 \%, 49.88 \%, 12.59 \%$ for symmetric case, respectively, and $68.92 \%$, $19.51 \%, 2.74 \%, 6.09 \times 10^{-7 \%} \%$ for asymmetric case, respectively, as shown in Figure S6d. From these results, asymmetric resistive switching synapse cell can reduce an undesired backward propagation with sneak current as compared to symmetric one. Nevertheless, asymmetric resistive switching synapse cell is also affected by a sneak current, it will be important problem to reduce the sneak current with backward propagation paths in neuromorphic device. For clear state separation, choosing state will be important problem in memristor based neuromorphic network because reading error is reduced if HRS1 state and HRS5 state are selected.

\section{References}

1. V.S. Dongle, A.A. Dongare, N.B. Mullani, P.S. Pawar, P.B. Patil, J. Heo, T.J. Park, T.D. Dongale, Development of self-rectifying $\mathrm{ZnO}$ thin film resistive switching memory device using successive ionic layer adsorption and reaction method. J. Mater. Sci.: Mater. Electron. 29, 18733-18741 (2018)

2. A.R. Poghosyan, E.Y. Elbakyan, R. Guo, R.K. Hovsepyan, Memristor memory element based on ZnO thin film structures, in Photonic Fiber and Crystal Devices: Advances in Materials and Innovations in Device Applications IX; 95861C (2015), pp. 5

3. A.S. Zoolfakar, R. Ab Kadir, R.A. Rani, S. Balendhran, X. Liu, E. Kats, S.K. Bhargava, M. Bhaskaran, S. Sriram, S. Zhuiykov, A.P. O'Mullane, K. Kalantar-zadeh, Engineering electrodeposited ZnO films and their memristive switching performance. Phys. Chem. Chem. Phys. 15, 10376-10384 (2013) 